\title{
Point de vue interne : ambiguïtés et transgressions
}

\author{
Suzette Ali \\ Université du Québec à Montréal
}

Selon les conventions de la narration personnelle, le narrateur $\mathrm{au}$ « je » ne peut être omniscient. Puisque l'histoire qu'il raconte comporte souvent des événements qu'il a vécus lui-même ou dont il a témoigné, elle ne peut contenir des informations qui dépassent les limites de ce qui peut être vu et connu par une personne. Cela n'est pas le cas des romans que nous étudions dans cet article: les narrateurs y affichent des connaissances profondes sur des faits importants des histoires qu'ils rapportent et auxquelles leurs compétences ne permettent pas d'habitude d'avoir accès. 
Dans Tarmac de Nicolas Dickner, le narrateur homodiégétique rapporte l'histoire de Hope, son amie, et de sa famille, dont tous les membres souffrent d'une obsession de la fin du monde. Atteinte à l'âge de la puberté de cette même obsession, Hope est entraînée dans des péripéties au fin fond du monde à la recherche du prophète qui a prédit la date de survenance de l'apocalypse. L'invisibilité du narrateur dans certains chapitres de ce roman et son omniscience non justifiée rendent problématique son existence au sein de l'histoire et nécessitent que nous nous y intéressions. Dans Gabrielle au bois dormant de Denyse Delcourt, les narrateurs sont tous dotés d'une omniscience irrégulière, ce qui rend peu crédible et même fantasque l'histoire qu'ils rapportent. Cette dernière tourne autour du meurtre présumé d'une adolescente, prénommée Gabrielle, par un étranger nouvellement installé dans le village. Si Jacqueline, l'amie intime de la victime, rapporte la majorité de l'histoire dans des chapitres séparés avec une précision parfois peu convaincante, les autres narrateurs comme Léo, Margueritte, etc., racontent, dans d'autres chapitres, certains événements que Jacqueline n'évoque pas avec la même précision.

Dans Dernier amour de Christian Gailly, qui raconte l'histoire d'un compositeur de musique contemporaine affrontant deux événements décevants (sa mort prochaine et l'échec de sa musique), le narrateur hétérodiégétique et omniscient deviendra au cours de l'histoire homodiégétique omniscient et se confondra par moments avec le personnage principal, Paul Cédrat. Cette pratique narrative rend ambiguë l'histoire racontée et déstabilise les conventions de la narration réaliste puisque le statut du narrateur n'est pas constant. 
Les trois romans affichent donc des entorses au point de vue interne qu'il s'agit d'analyser dans cet article. Avant d'examiner ces problèmes de narration homodiégétique, il nous faut définir le point de vue interne relié à ce type de narrateur. Afin de mieux cerner ce concept, nous examinerons les théories qui se sont consacrées à son étude. Celles-ci sont abondantes et appartiennent à diverses approches telles la narratologique et la linguistique et à différentes traditions, qu'elles soient française, tchèque et allemande. En effet, «la perspective narrative [...] a été, de toutes celles qui concernent la technique narrative, la plus fréquemment étudiée depuis la fin du XIXe siècle » (p. 190), écrit Gérard Genette. Divers termes ont été proposés par les théoriciens qui ont réfléchi sur ce concept : «focalisation», «point de vue», «aspect», «vision» ou « ocularisation », ce qui montre l'ambiguïté et la complexité qui entourent ses caractéristiques constitutives.

\section{Définition du point de vue interne}

Comme il a marqué la théorie narratologique et influencé toutes les recherches postérieures sur le point de vue, l'essai de Gérard Genette intitulé « Discours du récit » constituera le point de départ de cet examen théorique. La perspective représente pour Genette un des moyens qui permet de contrôler l'information présentée au lecteur. Le théoricien adopte le terme de «focalisation » dans son étude «[p]our éviter ce que les termes de vision, de champ et de point de vue ont de trop spécifiquement visuel» (p. 194), écrit-il. Le terme «focalisation», étant par ailleurs plus abstrait que le terme «point de vue», permet d'accentuer la distinction entre 
l'énonciation (ou la voix) et la représentation narrative (ou le mode). Grâce aux typologies proposées par Jean Pouillon et Tzvetan Todorov qui l'ont inspiré, Genette parvient à établir sa propre classification constituée de trois catégories: la focalisation zéro, la focalisation interne et la focalisation externe. La focalisation interne consiste à représenter ce que voit ou sait le personnage présent dans l'histoire : « le narrateur ne dit que ce que sait tel personnage » (p. 193). La focalisation interne signifie, chez Genette, que le point focal est situé à l'intérieur de la diégèse, puisque le personnage fait partie du monde fictif de l'histoire. Cette focalisation s'oppose, en cela, à la focalisation zéro, où le point focal est à l'extérieur du monde narré. Genette insiste sur l'importance de distinguer, au sein de la narration homodiégétique, entre le narrateur et le personnage qui constituent une même personne. Le narrateur, dans ce type de narration, possède un savoir sur l'histoire qui est naturellement plus profond que celui que possède le personnage, mais « [c]'est en général le "point de vue du héros" qui commande le récit, avec ses restrictions de champ, ses ignorances momentanées » (p. 205). Genette note, enfin, que «le parti de focalisation n'est pas nécessairement constant sur toute la durée d'un récit» (p. 196) et que «la distinction entre les différents points de vue n'est pas toujours aussi nette que la seule considération des types purs pourrait le faire croire » (p. 196-197). Il revient à la «focalisation interne» et signale que celle-ci «est rarement appliqué[e] de façon tout à fait rigoureuse. En effet, le principe même de ce mode narratif implique en toute rigueur que le personnage focal ne soit jamais décrit, ni même désigné de l'extérieur, et que ses pensées ou ses perceptions ne soient jamais analysées objectivement par le narrateur » (p. 197). Conscient donc qu'il est rare qu'une même 
focalisation domine complètement toute la surface du récit et que certaines infractions peuvent se manifester au sein des types purs, le théoricien introduit dans son étude deux catégories d'altérations: la paralipse et la paralepse. La première apparaît dans un récit à focalisation interne lorsque le narrateur donne moins d'information sur les pensées du personnage ou les événements vécus par ce dernier qu'il n'est nécessaire. La seconde peut aussi surgir dans un récit à focalisation interne lorsque le narrateur révèle les pensées des personnages présents dans l'histoire autre que «le personnage focal».

La classification du point de vue proposée par Genette constitue une base essentielle de la théorie narratologique. Pourtant, elle a été sujet à plusieurs reproches de la part des critiques et chercheurs. Rabatel signale, dans Une histoire $d u$ point de vue (1997), l'instabilité de la focalisation interne et identifie ce type de focalisation au seul monologue intérieur. Selon lui, la focalisation interne ne peut se manifester qu'à travers les discours intérieurs des personnages et non, comme l'explique Genette, à travers ce que le narrateur révèle de leurs pensées. Rabatel critique, de plus, la séparation entre le «mode » et la «voix», qui ne serait pas aussi catégorique que ne l'affirme Genette: «En dépit de certaines affirmations tranchées, il semble que Genette lui-même soit conduit, à plusieurs reprises, à relativiser, pour ainsi dire, la distinction entre mode et voix» (p.76). Rabatel note ensuite certaines analogies entre la narration hétérodiégétique et la narration homodiégétique. Le fait d'avoir la possibilité de présenter les événements suivant la focalisation zéro, interne ou externe dans le récit hétérodiégétique se présente aussi dans le récit homodiégétique, qui «fonctionne soit avec la focalisation zéro 
(le "je narrant" pouvant manifester son omniscience à l'égard des autres personnages ou à l'égard du personnage qu'il était autrefois), soit avec la focalisation interne (le "je narrant" adoptant la perspective limitée du “je narré”). C'est d'ailleurs en raison de ce parallélisme de fonctionnement que Genette envisage la possibilité d'une narration homodiégétique en focalisation externe » (p. 78). Bien que le degré d'informations diffère dans les deux cas de narration hétérodiégétique et homodiégétique, l'intention de Rabatel, à travers cette comparaison, est de montrer que l'acte de narration, lui, reste le même dans les deux types.

En outre, alors que Genette conçoit le point de vue comme une combinaison de la vue et du savoir du narrateur ou des personnages, Mieke Bal, qui part des travaux réalisés par ce théoricien, relie le concept du point de vue principalement à la sensation visuelle. Dans son étude Narratology: introduction to the theory of narrative parue en 1977 ainsi que dans son article «Narration et focalisation : pour une théorie des instances du récit» publié dans Poétique la même année, Bal revient sur certains concepts définis par Genette et les commente: « Si Genette confond certaines notions, sa typologie n'est pas sans valeur. Il est vrai qu'elle ne se laisse pas définir par la focalisation, terme qui reste à définir avec plus de précision, mais bel et bien par le savoir du narrateur » (p. 113). Elle relève certaines ambiguïtés dans la typologie du point de vue avancée par Genette, notamment en ce qui concerne la différence entre les sujets de la focalisation, d'une part, et les objets de la focalisation, d'autre part, que la typologie de Genette tend à occulter ou à amalgamer. Le sujet de la focalisation est différent dans les récits à focalisation zéro de celui des récits à focalisation interne : le narrateur omniscient est celui qui, dans 
le premier type, perçoit le monde représenté tandis que c'est le personnage qui voit le monde représenté dans le second type. Quant à l'objet de la focalisation, il est, lui aussi, différent dans les récits à focalisation interne et les récits à focalisation externe. Alors que le personnage dans le récit à focalisation interne est la personne qui voit, le personnage dans le récit à focalisation externe est, plutôt, celui qui est vu. Afin de combler ces quelques lacunes théoriques, Bal propose de distinguer la focalisation par un sujet (qu'il soit le narrateur ou le personnage) de la focalisation sur un sujet. Le premier cas concerne le focalisateur et le second le focalisé : «Le lecteur peut interpréter, voire juger un personnage. C'est que ce personnage, d'une manière ou d'une autre, est lisible, disons "visible" : le lecteur le voit. Le lecteur le voit par l'intermédiaire d'une instance, une instance qui voit et, en voyant, fait voir. Cette instance, que j'appellerai provisoirement le "focalisateur" [...] sera examinée dans la recherche de la réponse à la question "qui voit" »(p.115). Elle propose aussi de faire la distinction entre entre la focalisation interne et la focalisation externe, définies toutes les deux à partir de l'objet de la focalisation :

Le focalisé peut être visible ou invisible. J'entends par cette distinction la différence entre ce qui peut être perçu, par la vue, l'ouïe, l'odorat, le toucher, le goût, par un spectateur hypothétique, et ce qui ne peut pas l'être. C'est la distinction genettienne entre focalisation interne et focalisation externe, mais alors strictement réservée au focalisé. Faute de mieux, j'indiquerai par le mot "perceptible" ce qui relève de la présentation d'un focalisé extérieur, "imperceptible" est un focalisé qui peut être perçu uniquement de l'intérieur, comme l'analyse psychologique. (p. 120) 
Bal ajoute que le focalisé imperceptible n'est accessible que pour un narrateur ${ }^{1}$, qu'il soit hétérodiégétique ou homodiégétique. L'analyse de Bal, bien que complexe, tente de contourner les difficultés rencontrées par les catégories genettiennes. Elle a permis de mettre en lumière certaines distinctions manquantes dans la typologie de ce dernier et qui seront ensuite étudiées par d'autres chercheurs et critiques.

Jaap Lintvelt présente, à son tour, une étude détaillée du point de vue dans son Essai de typologie narrative : le "Point de vue»: théorie et analyse (1981). Malgré les ambiguïtés qui entourent le terme "point de vue », on remarque que Lintvelt se démarque de Genette et emploie ce terme dans sa typologie. En opposant les fonctions du narrateur à celles de l'acteur, ce théoricien arrive à dégager deux formes narratives de base : la narration hétérodiégétique et la narration homodiégétique. À partir de cette distinction, il établit trois types narratifs : le type narratif auctoriel et le type narratif actoriel, enfin, le type narratif neutre. Le type narratif constitue le centre d'orientation du lecteur et «est déterminé par la position imaginaire que le lecteur occupe dans le monde romanesque au plan perceptif-psychique, au plan temporel et au plan spatial» (p. 39). Lintvelt ajoute à ces trois plans le plan verbal parce que toute œuvre littéraire a pour fondement le langage. Puisque, dans la narration homodiégétique, la vie du personnage est perçue simultanément par le narrateur et par le personnage, nous nous limiterons à présenter seulement les types narratifs auctoriel et actoriel suivant les quatre plans introduits par Lintvelt. Le premier plan, perceptif-psychique, est associé à la

\footnotetext{
1 Mieke Bal utilise l'expression «focalisateur-narrateur» pour désigner le narrateur et le terme «focalisateur » pour désigner le personnage.
} 
perspective narrative, à la profondeur de cette dernière et au mode narratif. Suivant que le type narratif est auctoriel ou actoriel, le sujet de la perspective sera soit le personnagenarrateur soit le personnage-acteur. Cette distinction entre les sujets de la perception entraîne évidemment une variation de la profondeur de la perspective narrative. Celle-ci désigne la capacité d'un sujet à dévoiler ou non la vie intérieure d'un autre sujet que lui-même. Elle permet de distinguer entre focalisation interne et focalisation externe. Le personnage-narrateur «joui[t], en général, d'une perception externe/interne plus étendue que le personnage-acteur, tant par sa connaissance de l'issue de son histoire, que par une perspicacité accrue grâce à sa maturité. [...] [Mais] [é]videmment», ajoute le théoricien, «cette perception élargie est encore loin de l'omniscience du narrateur hétérodiégétique auctoriel » (p. 90). En effet, lorsque le personnage-narrateur épouse le point de vue du personnageacteur, il doit respecter la profondeur du savoir de ce dernier : la perception interne et externe du monde y sera donc limitée. La dernière catégorie du plan perceptif-psychique est le mode narratif: "Le narrateur», écrit Lintvelt, "peut présenter l'histoire selon deux modes narratifs, qui s'inspirent de la dichotomie platonicienne entre diégésis et mimésis » (p. 49). Le théoricien ajoute que le personnage-narrateur tend le plus souvent à présenter les événements de l'histoire sous forme de sommaire, au contraire du personnage-acteur, qui participe aux événements et qui ne peut les condenser en des sommaires. Le deuxième plan introduit par Lintvelt est le plan temporel. Grâce à ce plan, il est possible d'identifier «[d]eux relations temporelles [...] : le rapport entre la narration et l'histoire, que Genette range dans la catégorie de la voix, et la relation qui existe entre le récit et l'histoire » (p.52). La relation entre la 
narration et l'histoire joue un rôle important quant à la profondeur de la perspective narrative, en particulier l'ordre suivant lequel sont présentés les événements de l'histoire. Dans le type narratif auctoriel, la narration, qui est ultérieure, rend admissibles et certains les anticipations et les retours en arrière dans l'histoire racontée. Les anticipations certaines ne sont pas possibles avec le type narratif actoriel, car la narration y est simultanée aux événements vécus; seuls les retours en arrière y sont admissibles, lorsque le personnage-acteur évoque son passé. Le plan temporel implique aussi la durée qui influe sur la profondeur du savoir du narrateur. Le sommaire permet au personnage-narrateur de raconter une histoire dont la durée est longue, alors que la scène, associée au personnage-acteur, est de longueur temporelle réduite. Cela a pour effet de montrer la richesse des informations du personnage-narrateur sur l'histoire racontée par rapport au personnage-acteur, qui est incapable de saisir l'histoire dans sa totalité. Si le plan temporel joue un rôle important dans la profondeur de la perspective narrative, c'est aussi le cas du plan spatial, puisqu'il détermine les positions qu'occupent les sujets de la perception et, surtout, leur mobilité spatiale. Habituellement, il est impossible au personnage-acteur d'être omniprésent, mais cette restriction spatiale concerne aussi le personnage-narrateur et cela revient à sa nature humaine, qui l'empêche d'être présent partout. Malgré l'étendue du savoir de celui-ci, l'omniscience et l'omniprésence lui sont inaccessibles. Le dernier plan, qui se rapporte au langage, traite du statut du narrateur et de ses fonctions. Moins relié à la perspective narrative que les autres plans, ce plan ne sera pas traité ici.

Le problème de délimitation du point de vue est étudié par Pierre Vitoux dans son article «Focalisation, point de vue, 
perspective», publié dans la revue Protée en 1988. Vitoux commence son article par une critique de l'analyse genettienne de la focalisation. En effet, il reproche à Genette sa conception de la focalisation interne, définie à partir de l'acte de restriction du champ visuel ${ }^{2}$ : «Il associe, d'une part, la notion que le narrateur a, au départ et en tant que tel, une vision non focalisée qui lui permet de pénétrer dans le for intérieur d'un de ses personnages - et, d'autre part, à partir de là la possibilité de se limiter à la connaissance de l'histoire que peut avoir ce personnage, c'est-à-dire à une vision perceptuelle ou externe, qui aboutira donc entre autres choses à une connaissance interprétative et non intuitive d'autrui, de l'autrui de ce personnage» (p.34). Afin d'éclaircir le concept de la focalisation interne, Vitoux propose de distinguer, d'une part, entre « le sujet de la focalisation, identifiable au départ avec le narrateur, mais avec possibilité de transfert - et l'objet de la focalisation, qui peut lui-même être de deux types différents, selon qu'il est perçu uniquement de l'extérieur (focalisation objet externe) ou qu'il est perçu de l'intérieur par un accès à la conscience d'un personnage (focalisation objet interne) » (p. 34); et d'autre part, entre deux types de focalisation-sujet : la première est la Focalisation-sujet non déléguée (Fs nd), où le narrateur est lui-même le focalisateur sans qu'il y ait restriction de champ ; la seconde est la Focalisation-sujet déléguée (Fs d), dans laquelle le narrateur cède le centre de focalisation au personnage, mais en gardant sa fonction de narration. Vitoux explique ensuite la relation du sujet à l'objet qui peut

\footnotetext{
${ }^{2}$ Pour présenter ce que le personnage est en train de percevoir, le narrateur réduit son champ visuel à celui de ce personnage. La focalisation se situe, suite à cela, dans le personnage. Genette désigne ce mouvement par l'expression de « restriction du champ ».
} 
commencer par être simple et objective (Fs nd $\rightarrow$ Fo ext), telle la description d'un décor ou d'un personnage, pour ensuite devenir psychologique (Fs nd $\rightarrow$ Fo int) et permettre de rendre compte des pensées d'un personnage, pour enfin devenir interne en déléguant la focalisation au personnage (Fs d) : "Autrement et simplement dit, il faut d'abord "voir dans" pour ensuite "voir avec"; et inversement le fait de découvrir les pensées d'un personnage nous révèle du même coup la façon dont il voit les choses [Fo int $\rightarrow$ Fs d]» (p. 35). La Fs d ne permet donc pas un savoir profond de l'objet: «Un personnage», écrit Vitoux, «a pour définition d'avoir une existence fictive dans une histoire, et d'être par conséquent soumis à la condition réaliste ou pseudo-existentielle d'avoir une connaissance purement perceptuelle du monde et des hommes. En particulier, il ne peut pas pénétrer les pensées des autres, il ne peut que les inférer à partir de ce qui lui est perceptible: leurs actions, leurs paroles, ce qu'en disent les autres personnages soumis à la même limitation» (p. 33-34). Cette première analyse de Vitoux concerne la focalisation. Or, comme l'indique le titre "Focalisation, point de vue, perspective », ce critique cherche à discerner, dans son article, les caractéristiques spécifiques de chacun de ces termes même s'ils sont synonymes. Si la focalisation concerne la quantité et le type d'information (externe, interne) de la vision, le point de vue s'applique, lui, à la qualité de l'information fournie au lecteur «qui fait que, par exemple, les focalisations à partir de deux situations comparables ne convergent pas nécessairement pour nous donner une image unique et cohérente » (p. 36). Par conséquent, dans les situations où le narrateur est le seul focalisateur (Fs nd), l'information présentée par ce dernier constitue le plus souvent une vérité incontournable. Mais cela 
n'exclut pas la présence de certains problèmes de «fiabilité » $\mathrm{du}$ narrateur dans certains textes, et cela, à cause de la subjectivité du narrateur-focalisateur. Lorsque le narrateur délègue la focalisation au personnage (Fs d), les informations données sont souvent personnelles et peu profondes. La séparation entre la focalisation du narrateur et la focalisation du personnage diminue donc la consistance des événements rapportés. Quant à la perspective ${ }^{3}$, elle émane du sens général du texte. Elle découle, en effet, des différents points de vue qui existent dans le texte, mais aussi de la relation de ce texte avec l'univers extra-textuel, plus précisément culturel. Selon Rabatel, la théorie de Vitoux sur la délégation de la focalisation par l'instance narrative à un personnage est plus convaincante que les théories qui l'ont précédée. Ce dernier trouve, en effet, qu'elle résout le problème du savoir illimité du narrateur, puisque la focalisation sujet non déléguée (Fs nd) peut s'appliquer autant à la focalisation objet interne (Fo int.) qu'à la focalisation objet externe (Fo ext.). Le narrateur n'est plus conçu d'emblée comme une personne omnisciente. Mais, en même temps, Rabatel ne s'accorde pas avec Vitoux sur la restriction de la focalisation déléguée à un personnage à la seule focalisation externe de l'objet. Selon lui, il est possible au personnage de comprendre les pensées d'autrui en interprétant ses mimiques et ses gestes.

En conclusion à cette présentation théorique du point de vue du narrateur homodiégétique, il nous faut indiquer que, d'une étude à l'autre, on s'entend à reconnaître que la profondeur de la perspective narrative du narrateur

\footnotetext{
${ }^{3}$ Vitoux n'entend pas la perspective à la manière de Genette et des autres théoriciens de la narration. Il réserve ce terme au message que le texte cherche à transmettre aux lecteurs plus précisément, au sens général du texte.
} 
homodiégétique reste toujours limitée. Même si le narrateur jouit d'une supériorité sur le personnage, il ne peut tout savoir ni tout contrôler parce qu'il constitue lui-même un personnage. Cela explique le choix des romanciers réalistes de recourir au récit à la troisième personne, qui permettait l'accès aux pensées de tous les personnages présents dans le récit sans que cela ne détruise les lois de la vraisemblance. Le privilège du point de vue omniscient accordé au narrateur hétérodiégétique est traditionnellement refusé au narrateur homodiégétique, même si cette exigence n'est pas respectée dans les romans contemporains qui nous intéressent. Dans ces romans, le narrateur homodiégétique est doté de connaissances qui transgressent les limites de la vraisemblance. Il constituera pour cette raison l'objet de nos prochaines analyses. Une dernière précision à ajouter concerne notre choix pour le terme «point de vue» parmi les divers termes proposés par les critiques et théoriciens dans l'analyse de la perspective narrative qui suit. Cela revient à la capacité de ce terme de signifier en même temps la vue et la façon de penser, c'est-àdire l'opinion et, plus largement, le savoir.

\section{Les narrateurs homodiégétiques dans Tarmac, Gabrielle au bois dormant et Dernier amour}

Les romans que nous étudions constituent des récits personnels dans lesquels les narrateurs révèlent certaines expériences qu'ils ont eux-mêmes vécues ou dont ils veulent témoigner. Étant impliqués dans les deux niveaux qui forment le récit, soit la narration et l'action, ils nous communiquent leurs émotions et leurs jugements par rapport aux événements relatés à partir de deux points de vue différents : le premier est lié à leur statut 
de narrateurs ou au point de vue du je-narrateur et le second se rapporte à leur statut d'acteurs ou au point de vue du jepersonnage. Notre but, dans cette partie de l'article, est d'analyser ces deux types de points de vue afin de montrer les convergences et les divergences qu'ils présentent par rapport aux exigences habituelles de la narration homodiégétique. Il ne faut pas oublier que, lorsqu'un narrateur homodiégétique relate les événements selon son propre point de vue, des préjugés, des fantasmes et des mensonges peuvent se mêler à son histoire : «Une histoire peut ou bien informer le lecteur, ou bien le désinformer » (p. 498), écrit Michal Glowinski. Le seul capable de jouer de la véracité de l'histoire rapportée est le narrateur. C'est son point de vue qui importera le plus dans l'analyse qui suit, sans que cela ne nous empêche d'examiner son point de vue d'acteur. Cependant, dans un premier temps, nous tenterons de séparer les deux perspectives narratives, bien que cela soit moins facile dans les récits homodiégétiques à cause du va-et-vient constant entre le point de vue du je narrateur et le point de vue du je acteur.

Dans Tarmac de Nicolas Dickner, le «je» narrant n'apparaît qu'à la fin du roman : on suit, par conséquent, dans presque la majorité du récit, le point de vue du je-narré. Autrement dit, le foyer de la perception est situé dans le regard de Michel Bauermann adolescent. Il devient donc plus important d'observer, dans ce roman, le point de vue du personnage que le point de vue du narrateur. En appliquant les formules par lesquelles Pierre Vitoux a représenté le point de vue (ou la focalisation selon ses propres mots), on pourra représenter le point de vue $d u$ «je» personnage par Fs $d \rightarrow$ Fo ext. avec certaines transgressions qui seront explicitées plus loin, et le point de vue du narrateur par 
Fs nd $\rightarrow$ Fo int avec là aussi des transgressions qui seront étudiées ultérieurement. Il faut se rappeler que, dans ce roman comme dans tout roman, il y a une différence entre la personne qui parle et la personne qui voit, différence qui est au cœur de la théorie narratologique genettienne. C'est le narrateur adulte qui parle dans Tarmac, depuis le début du récit jusqu'à la fin, même si le « je » est adolescent durant presque toute l'histoire. C’est donc le « je » personnage qui perçoit les scènes puisqu'il est situé à l'intérieur de l'histoire.

La même différence entre mode et voix touche le roman Gabrielle au bois dormant. Dans ce roman, le je-narrant ne prend pas vraiment la parole, mais le lecteur a accès aux pensées de Jacqueline, la narratrice, et apprend la grande partie de l'histoire à partir du point de vue de cette dernière. La présence des photos et des images au début du roman le confirme: «l'irruption de ces images qui, pourtant enfouies en moi depuis longtemps, n'avaient en rien pâli » (Delcourt, p. 9). La narratrice revoit mentalement les scènes de son enfance, exercice de remémoration propre à son statut de narratrice. Pourtant, le point de vue du je-narré se détache graduellement de ce point de vue dominant et l'on retourne au passé de la narratrice pour revivre avec elle, en tant que personnage, les événements de son enfance : «Le narrateur ne raconte pas par la grâce d'une bonne mémoire, il voit le passé comme présent grâce à une faculté plus qu'humaine » (p. 74), écrit Wolfgang Kayser. En effet, les scènes reliées aux souvenirs d'enfance et qui sont perçues par le je-narré de Jacqueline sont rapportées de manière immédiate, ce qui donne l'impression qu'elles sont simultanées à l'acte de narration. Or, malgré la différence entre les deux points de vue, il y a similitude dans la profondeur du savoir de ceux-ci. Le je-narrant est âgé de 50 ans et montre plus 
de maturité que le je-narré âgé de 16 ans sans toutefois témoigner de plus de connaissances sur l'histoire racontée: « Oh! Inutile de revenir là-dessus, vraiment. Il vaut mieux si possible de s'engager à croire à la pureté du présent » (Delcourt, p. 16), pense-t-elle sur la mort de Gabrielle. S'ajoute à cette situation narrative la variabilité du sujet focal. Le point de vue n'est pas limité à un seul personnage, mais s'étend alternativement à plusieurs autres. L'intérêt de leur présence réside dans le rapport qu'ils entretiennent avec le point de vue dominant de Jacqueline. S'ils se complètent tous, cela aura pour effet de rendre vraisemblable l'histoire de la narratrice, mais s'ils s'opposent au point de vue dominant de cette dernière, celle-ci pourrait être considérée comme non fiable. Bien que rien dans le texte n'indique l'âge de ces narrateurs, il est bien clair qu'une certaine distance temporelle sépare les «je» narrant des « je » narrés. Sauf que cette distance temporelle ne joue pas vraiment un rôle déterminant par rapport à la narration des faits, car la grande majorité de l'histoire est racontée par Jacqueline.

Enfin, Dernier amour manifeste des jeux sur le point de vue d'égale importance aux romans précédents. Cependant, à la différence des autres romans, le « je » narrant n'y forme pas une seule personne avec le « je » narré. Comme nous l'avons vu plus haut, dans Tarmac et dans Gabrielle au bois dormant, les « je » narrants et les «je » narrés renvoient toujours à une même personne, qui est en même temps le narrateur et le personnage principal. Cela n'est pas le cas de la situation narrative dans Dernier amour. Le «je» narrant représente, en effet, un narrateur anonyme et le «je » narré représente le personnage de Paul Cédrat. Ensuite, alors qu'au début de ce roman, on se situe à l'extérieur de l'histoire à cause de la position 
extradiégétique du narrateur hétérodiégétique, les choses changent lorsque le point de vue de ce narrateur vient se confondre avec le point de vue du personnage. L'on se trouve transporté au sein de l'histoire sans pour autant être séparé du point de vue du narrateur. Les deux points de vue à considérer sont donc le point de vue du narrateur puis le point de vue du narrateur devenu personnage. La notion de restriction du champ de Genette est fondamentale dans ce roman. Il faut comprendre, en effet, que le narrateur ne réduit pas son point de vue au point de vue de son personnage, mais se réduit luimême en ce personnage, d'où les fluctuations des points de vue. Le point de vue du narrateur devenu personnage épouse le point de vue du personnage et l'on n'arrive plus à distinguer entre ceux-ci. Ce premier travail de description des sujets de la perception dans les quatre romans conduit à l'examen de l'objet de la perception. Dans les lignes qui suivent, nous aborderont cet aspect en analysant ce que ces narrateurs homodiégétiques sont capables de voir et ce qu'ils sont capables de savoir.

Le savoir du narrateur homodiégétique est habituellement considéré comme problématique puisque relatif: "Ainsi, le roman à la première personne", explique Michal Glowinski, « est un mode de récit très spécifique, dans la mesure où le fait pour le narrateur de posséder un savoir ou d'en être privé est d'égale importance. Et le savoir lui-même est problématique » (p. 503). Puisque le point de vue de ce type de narrateur est restreint, son savoir est partiel et son récit est souvent jugé moins exhaustif que le récit du narrateur hétérodiégétique. Comme ils sont homodiégétiques ou autodiégétiques, on s'attend à ce que les narrateurs qui existent dans ces romans connaissent leur propre histoire de vie, car leur rôle est effectivement de se raconter. En d'autres termes, 
ces narrateurs ne font que raconter des histoires qui les concernent; il va de soi donc qu'ils les maîtrisent bien. Cependant, Michel Bauermann dans Tarmac joue plutôt le rôle de témoin dans sa propre histoire. Malgré l'abondance des descriptions et des faits divers provenant d'un peu partout dans le monde, ce narrateur ne nous renseigne pas sur sa vie privée : «Journée de travail typique, passons les détails», dit-il pour décrire sa vie actuelle (Dickner, p. 256). Toute son attention est tournée vers son amie Hope et ses aventures. Pour cette raison, son savoir s'oppose déjà au savoir des narrateurs homodiégétiques normalement réduit aux pensées et à la vie intérieure du je-narrant et n'excédant fort peu la sphère du familier. De plus, lorsque ce type de narrateur n'a pas été témoin de ce qu'il rapporte, il explique généralement d'où il tient ses informations, ce qui ne s'applique pas au narrateur du roman de Dickner : la profusion des détails, des informations et des descriptions sur la vie de Hope Randall que le narrateur mentionne n'est jamais justifiée. Ainsi, dès les premières pages, le roman se rapproche plus d'un récit hétérodiégétique que d'une histoire personnelle.

Qu'un individu trouve difficile de se comprendre ou d'analyser des événements qui lui arrivent n'a rien pour heurter le sens commun. Lorsque les événements font partie du passé, il est plus facile d'y retourner et de les interpréter. C'est pour cette raison que les récits de soi constituent souvent des narrations ultérieures. Le narrateur profite du recul avec les événements vécus afin de rectifier ses informations à leur sujet. Le savoir du je-narrateur devient, à la suite de cette distance temporelle, supérieur au savoir du je-personnage et son point de vue devient plus étendu. Sur ce point, le roman Gabrielle au bois dormant constitue une exception. Dès le début du roman, la 
narratrice Jacqueline se montre plus informée non seulement sur ce qui se rapporte à sa vie passée, mais aussi sur tout ce qui se rapporte aux faits présents: "Nous n'en avons pas encore parlé non plus, mais nous l'avons tous intimement reconnu, j'en suis sûre », affirme-t-elle (Delcourt, p.11). Ces connaissances élargies, au début du récit, restent pourtant explicables. Dans bien des récits, le narrateur révèle dans l'incipit beaucoup d'informations en se présentant ou en présentant ses personnages, et cela, dans le but d'expliciter les circonstances de son histoire. Dans le cas de Jacqueline, on pourrait dire que toutes les informations qu'elle révèle sur les autres narrateurs constituent des opinions personnelles, surtout que l'on se situe dans ses pensées à cette étape de l'histoire. Ainsi, après tant d'années d'amitié, il est logique que la narratrice puisse prévoir certaines choses sur ses amis, d'où cette profondeur de son savoir toujours vraisemblable et justifiable : "Suzanne restera immobile, je le sais, jusqu'à ce que son esprit se remplisse à nouveau d'événements minuscules qu'il lui faudra vite exposer avant qu'ils ne disparaissent», dit-elle (Delcourt, p. 13). Son savoir provient du fait qu'elle connaît bien tous les problèmes que vivent les autres narrateurs : «Il ne m'est pas nécessaire d'entendre les paroles qu'il prononce » (Delcourt, p. 14), révèlet-elle à propos de Paul, elle qui sait la vérité cachée de ce médecin : "Derrière le masque du Bon Docteur se trouve un autre Paul, celui-là furieusement agité, souffrant. [...] En effet, qu'y a-t-il de commun entre le médecin consciencieux et celui qui se drogue avant de sortir, la nuit, dans les bars gays les plus durs de la ville? » (Delcourt, p. 15) Il faut aussi tenir compte du fait que Jacqueline joue le rôle de témoin dans cette histoire et qu'il existe une importante différence de point de vue entre le narrateur homodiégétique et le narrateur autodiégétique. Le 
premier point de vue reste toujours externe, le narrateurtémoin ne pouvant raconter que ce qui lui est perceptible selon les propositions de Genette, alors que le second est interne, le narrateur-protagoniste étant capable de nous renseigner sur ses propres sentiments et pensées en plus de nous renseigner sur ce qu'il observe. Même si le narrateur-témoin exprime ses sentiments et pensées par rapport à ce qu'il voit, ses impressions personnelles ne seront pas aussi nombreuses et importantes que s'il était un narrateur autodiégétique. Le point de vue de Jacqueline devrait être donc externe et non pas interne $^{4}$, car, dans ce roman, elle ne fait que rapporter ce qu'elle observe. Cela justifie en quelque sorte sa tentative d'élargir son savoir par des connaissances intuitives dans la mesure où elle cherche à comprendre ce qui se passe autour d'elle. Lorsque Léo devient le narrateur au chapitre $\mathrm{V}$, il est lui aussi doté de connaissances élargies sur son entourage. Ce savoir profond quand il se présente au début du roman est lui aussi acceptable puisque justifiable : «Devant moi, je sens Jacqueline se raidir, prise de panique. Je voudrais être loin d'ici, je voudrais vite rentrer, le cour me bat, au secours, je sombre, pense-t-elle » (Delcourt, p. 36). L'omniscience provient d'un sentiment ou d'une impression que Léo découvre sur le visage de son amie. Quant à Marguerite, cette narratrice est à son tour pourvue d'un savoir intime sur ses amies: "Debout sur le quai, éclatante dans un deux-pièces aux reflets métalliques, Thérèse contemple elle aussi ce spectacle. C'est parfait! pense-t-elle. Tout se passe comme prévu! Ses invités se divertissent, parfait! » (Delcourt, p. 52-53)

\footnotetext{
${ }^{4}$ Les termes externe et interne sont utilisés dans cette phrase dans le sens donné par Genette.
} 
Enfin, dans Dernier amour, le narrateur n'est pas d'emblée homodiégétique, comme dans les autres romans, mais supérieur à son personnage et absent de l'histoire qu'il raconte, donc hétérodiégétique : «On a vu. Surtout entendu mais aussi vu. La salle se déchaîner contre la musique de Paul » (Gailly, p. 14), annonce-t-il. L'utilisation du pronom indéfini «on» instaure un point de vue objectif qui caractérise souvent les narrateurs hétérodiégétiques. Le narrateur est aussi omniscient à la façon d'une instance abstraite libérée des limites du narrateur personnel. Il est capable de connaître les sentiments et les pensées des personnages: "Son cœur bat très fort" (Gailly, p. 12), dit-il, puis révélant les pensées intimes de son personnage, il ajoute : «Comment vont-ils me recevoir? Quel accueil pour moi? Qu'est-ce qu'on me réserve? Il n'allait pas tarder à le savoir» (Gailly, p. 14). Mais cette supériorité du narrateur changera au cours du roman lorsque le point de vue de ce dernier s'unira au point de vue de son personnage; le lecteur pourra alors, grâce à cette transposition de point de vue, vivre et éprouver les événements comme le narrateur semble les éprouver, lui-même, à travers Paul Cédrat. Grâce à la fusion des points de vue, la narration se rapprochera dans ce roman de la présentation directe.

Cette première analyse des différents savoirs des narrateurs que l'on trouve au début des romans montre que ces derniers respectent les conventions de la narration homodiégétique (et hétérodiégétique dans le cas de Dernier amour), entre autres les limites de leur point de vue interne, tant qu'ils n'ont pas encore entrepris le récit de leur enfance. Le retour dans le passé bouleversera, toutefois, le point de vue 
interne et poussera les narrateurs à dépasser leurs facultés humaines pour comprendre des événements passés qu'ils n'ont pas pu juger ni comprendre malgré leur maturité et le passage du temps.

\section{Narrateurs homodiégétiques omniscients}

Lorsque le « je » raconte des souvenirs passés, il lui est possible d'être omniscient par rapport à sa propre histoire. Ses connaissances supérieures sont considérées comme vraisemblables, car certaines informations qu'il ignorait pendant le déroulement des événements peuvent lui être révélées plus tard. Mais le fait d'avoir accès à toute information concernant le passé et d'avoir des connaissances illimitées sur l'histoire racontée rend problématique la situation narrative, en particulier la profondeur du point de vue interne dans les romans de notre étude. Ces problèmes liés à la vraisemblance de la narration apparaissent lorsque les « je », dotés d'un point de vue omniscient, racontent des histoires qui dépassent leur savoir ou rapportent des informations qu'ils ne sont pas censés savoir. Même s'ils justifient leur savoir, ce que fait Jacqueline dans Gabrielle au bois dormant en faisant croire que les informations qu'elle tient lui ont été révélées par un autre personnage, les connaissances qu'ils déploient dépassent toute tentative de légitimation. Dès lors, même si Jacqueline affirme : "Personne d'autre que moi ne savait qu'elle était sortie. Lorsque Gabrielle avait quitté la chambre, ils dormaient tous d'un sommeil calme et profond » (Delcourt, p. 9), et même si elle maintient toute sorte d'excuses pour justifier son savoir : «J'y étais, j'ai tout vu » (Delcourt, p. 14), nombre d'informations 
semblent être connues par cette narratrice d'une manière indéterminée, comme si elle possédait les capacités d'un narrateur omniscient. " La tête appuyée contre son dos, elle se laissait un moment bercer par le trot de la bête. L'odeur musquée de sa peau, la ligne pâle de son cou qu'elle caressait du bout des lèvres, son corps souple entre ses bras, tout était délicieux [...]. » (Delcourt, p. 86) : c'est ainsi qu'elle décrit une scène d'amour entre Walter Black et Gabrielle à laquelle elle n'a jamais assisté. Cela confirme l'appropriation par cette narratrice de compétences qui transgressent les limites de sa nature humaine. C'est aussi le cas des autres narrateurs présents dans ce même roman et dans les autres romans choisis. Leur point de vue interne devient omniscient, présentant des informations illimitées sur leur monde. Notons, par exemple, cette information livrée par le narrateur Michel Bauermann de Tarmac au sujet de Hope : "Hope hocha la tête d'un air décidé, jugea sage de ne rien ajouter. » (Dickner, p. 153) Même si, dans ce roman, on passe de la narration homodiégétique à la narration hétérodiégétique et que l'omniscience ne provient pas immédiatement du narrateur homodiégétique, il est toujours très clair que c'est le même narrateur qui continue à parler dans la suite du récit. Ces quelques exemples illustrent les entorses à la profondeur du point de vue présentes dans nos romans. Dans les lignes qui suivent, nous nous arrêterons aux types d'information que les narrateurs des romans choisis sont capables de nous révéler.

Parmi les informations que nous livrent les narrateurs homodiégétiques et qui ne pourraient être connues de ces derniers que s'ils possédaient les capacités du narrateur omniscient se trouvent les pensées d'autrui. Dans Tarmac, cette capacité d'accéder aux réflexions d'autrui est une des 
compétences de Michel Bauermann. Lorsque son amie part aux États-Unis puis au Japon, ce narrateur n'est pas seulement capable de la suivre partout, mais il glisse dans ses pensées pour nous les dévoiler : "C'est bon à savoir", songea Hope » (Dickner, p. 161) et «Pendant une seconde, Hope imagina ses rognures d'ongles envoyées sur une planète vierge et sans vie, comme la Terre à l'époque de la grande soupe primitive » (Dickner, p. 177). Cela montre son omniscience, soulignée d'ailleurs de façon ostentatoire par les petits détails. L'omniscience est accordée aussi aux narrateurs de Gabrielle au bois dormant: "Je maquille une poupée de chiffon, pense tristement Gabrielle » (Delcourt, p. 126), révèle Jacqueline. Tout comme elle l'est à celui de Dernier amour, dont les compétences de narrateur hétérodiégétique de ce roman ne sont jamais abandonnées même lorsqu'il devient homodiégétique et s'introduit au sein de l'histoire à l'aide d'un « je » : «Elle était pressée, [...]. Avait médité la chose toute la matinée. Ne parvenait pas à se décider. N'osait pas. Avait peur. Je comprends ça » (Gailly, p. 91). Les romans offrent donc tous des exemples de transgression du point de vue interne, car, comme l'explique Jaap Lintvelt, "[q]uand le narrateur adopte une position temporelle interne, il se situe dans le présent d'un personnage romanesque, si bien que son point de vue est "synchrone" avec celui de ce personnage (cf. type narratif actoriel)» (p.171). Avec Dernier amour, l'omniscience du narrateur atteindra même les pensées des lecteurs : «Je parle de la boîte de vitesses. Je précise pour ceux qui n'y connaissent rien. Les autres avaient compris » (Gailly, p. 48), "Se tourna vers le piano. Elle l'avait repéré. Vous pensez bien » (Gailly, p. 93-94), dit-il. En lisant de la sorte dans les pensées des «lecteurs», le narrateur semble sortir du cadre fictif de 
l'histoire et revenir à la situation de communication avec le lecteur.

En plus de l'accès aux pensées des personnages, l'omniscience des narrateurs est confirmée par la richesse des informations présentées sur les histoires racontées. Dans Tarmac, le savoir du narrateur augmente progressivement à partir du chapitre 3, sans faire montre encore d'omniscience. La profusion des précisions et des détails semble indiquer que le narrateur a acquis beaucoup d'informations sur l'histoire de Hope. Au chapitre 49, cependant, le narrateur occupera brutalement le statut de narrateur hétérodiégétique omniscient. Ce changement imprévu de point de vue lui permet de raconter les aventures de Hope à l'étranger sans y avoir assisté : «Au coin Lafayette, elle fila un billet à un clochard dont le carton annonçait "The End Is Near"» (Dickner, p.150). L'intérêt qu'il prête aux indications spatiales et aux descriptions confirme son omniscience et son rôle d'observateur : Hope « en profita pour examiner la réception. Quelques chaises, un bureau, un corridor - et, surtout, un grand cadre de Charles Smith : un individu en chemise médiévale blanche, avec sourcils de Zeus et regard perçant» (Dickner, p. 152). La description minutieuse de tout ce que Hope voit, entend et rencontre à New-York et au Japon finit par devenir invraisemblable: «Comment le narrateur sait-il ce qui se passe au Japon puisque Hope ne le contacte plus après son départ des États-Unis? Le récit qu'il fait de sa vie au Japon est peu vraisemblable du point de vue de la logique narrative, mais l'invraisemblable fait aussi partie intégrante du récit; d'ailleurs, depuis qu'elle était au Japon, Hope "se découvrait [...] une capacité croissante à tolérer l'invraisemblable et l'improbable" (186) » (p. 124), écrit Pascal Riendeau. La même chose se reproduit dans Gabrielle au bois 
dormant et dans Dernier amour, la multiplicité des descriptions et des informations montre à la fois l'omniscience des narrateurs homodiégétiques et l'invraisemblance de leur savoir : "C'est une grosse dame assise sous un parasol de la taille d'une ombrelle. Elle tricote un pull jacquard pour son grand fils » (Gailly, p.66-67), note le narrateur dans Dernier amour. L'information pourrait sembler anodine si la dame décrite n'était pas un personnage secondaire et anonyme. On a donc parfois l'impression d'acquérir une multitude d'informations qui ne concernent pas 1'évolution des histoires et servent surtout à signaler avec ostentation le savoir extravagant des narrateurs.

D'autres types d'informations révèlent l'omniscience des narrateurs de façon plus spécifique à chacun des romans. Le narrateur de Tarmac est omniprésent. Michel Bauermann donne l'impression de survoler le monde et le souligne d'ailleurs : « je flottais hors de mon corps, quelques mètres audessus de la scène » (Dickner, p. 260). L'élévation lui permet de surplomber plusieurs endroits et plusieurs pays. La capacité de communiquer avec l'au-delà dans Gabrielle au bois dormant est le signe d'un pouvoir supérieur que possèdent les narrateurs. Marguerite, Léo et Jacqueline arrivent à entendre le fantôme de leur amie : «En ce moment même ondulant dans l'eau du lac, demandant demandant demandant, l'ombre appelle... Léo et Jacqueline l'ont entendue, et leur désir s'est refroidi tout à coup » (Delcourt, p. 53-54). Ces derniers ont accès à un monde dont est privé le reste des personnages et la récurrence d'une telle interaction vient renforcer leur omniscience. Dans Dernier amour, ce sont les diverses digressions qui envahissent ce roman et mettent ainsi en lumière l'omniscience du narrateur homodiégétique: "J'aurais dû lui dire de rouler droit devant 
lui. Jusqu'à ce que je vous dise de vous arrêter. Ou bien jusqu'à ce que je n'aie plus d'argent pour vous payer » (Gailly, p. 41), y trouve-t-on par exemple. Le narrateur invente quelques bouts de récits, ce qui montre sa liberté de conduire l'histoire comme il veut. L'omniscience dans Dernier amour renvoie donc au pouvoir de fabuler du narrateur. Bref, tous les narrateurs des quatre romans choisis sont omniscients. Ils voient et entendent au-delà de ce que leurs facultés humaines devraient leur permettre normalement de faire. La focalisation interne est par conséquent transgressée. Comme nous l'avons vu dans la partie théorique, en narration homodiégétique le narrateur qui s'exprime en employant le «je » est personnalisé. Étant une personne, il ne peut s'attribuer des pouvoirs surhumains sous peine de détruire le réalisme de l'histoire. II peut deviner les pensées des autres, les déduire, et non les exprimer avec certitude à la manière d'un narrateur omniscient. Il ne peut non plus raconter ce qui s'est passé hors de sa présence comme s'il y avait assisté. Cela n'empêche évidemment pas le roman homodiégétique de commettre ponctuellement des infractions à l'égard de ces principes, notamment en ce qui concerne le point de vue interne. Comme nous l'avons mentionné plus haut, Genette désigne les transgressions du point de vue par le terme d'« altérations » et les infractions du point de vue interne par le terme de «paralepses». Celles-ci surviennent lorsque le narrateur dévoile plus d'informations que ne le lui permet son point de vue restreint. Comme l'explique à son tour François Jost, « [l]a paralepse véritable ne commence qu'au moment où le récit change de foyer cognitif sans justification (comme lorsque le narrateur homodiégétique de la Recherche pénètre dans la tête de Bergotte pour nous livrer ses dernières pensées) » (p. 20). Ces altérations sont néanmoins temporaires 
et le point de vue principal doit rester dominant, selon ces deux critiques. Or, sur ce point aussi, les trois romans étudiés rompent avec les types de transgressions identifiés de la narration homodiégétique, car l'appropriation des narrateurs de certains pouvoirs supérieurs n'est pas temporaire. $\mathrm{Au}$ contraire, on remarque que le point de vue omniscient s'y impose autant que le point de vue interne. Cette double position n'est pas possible dans le roman réaliste classique, qui a attribué au narrateur «je» un savoir limité et rattaché l'omniscience au seul narrateur hétérodiégétique, même si la narration omnisciente est un subterfuge de la fiction. On pourrait bien sûr objecter que, dans le monde de la fiction, tout est possible et que rien en théorie n'empêche le narrateur personnel de posséder un savoir illimité sur l'univers qu'il raconte. Autrement dit, de même que le narrateur hétérodiégétique possède un point de vue omniscient, de même le narrateur homodiégétique pourrait adopter en partie ce point de vue, s'en servant comme d'un moyen heuristique pour tenter de combler les lacunes de son savoir et de ses perceptions.

On pourrait, en effet, avancer que le recours à une forme d'omniscience vient combler un manque de savoir et un manque d'assurance. Grâce à l'omniscience, l'accès aux connaissances du passé paraît réalisable. La volonté de contrôler le réel se manifeste dans le roman de Dickner, Tarmac. Le narrateur confesse avoir commis une erreur en laissant Hope partir. S'il ne l'avait pas laissée s'éloigner, sa vie aurait été différente : «Le rire se coinça dans ma gorge. Je mesurais soudain, brutalement, l'ampleur de mon erreur. J'avais laissé Hope filer à l'autre bout du monde sans réagir, comme un idiot, alors qu'il aurait fallu la poursuivre, la 
convaincre de rebrousser chemin - ou alors disparaître avec elle» (Dickner, p. 243). Dans Tarmac, l'omniscience et l'imagination servent au narrateur à disparaître avec Hope, autrement dit à être avec elle sans être là. Imaginaire et réel se rencontrent aussi dans Gabrielle au bois dormant, mettant en évidence la contribution de l'imagination dans la compréhension et la recréation du réel : «Marcher, marcher jusqu'à l'aube parmi des fantômes en quête de l'époux qui, sans doute, l'attend quelque part, mais où? où? où donc? Ainsi, j'imagine, se passa pour Gabrielle la première nuit de la longue attente» (Delcourt, p.159). Cela peut être comparé aux possibles narratifs présents dans Dernier amour. Alors que, dans Gabrielle au bois dormant, l'omniscience vise à comprendre et à transformer le réel, dans Dernier amour, l'omniscience constituerait plutôt un refus du réel et un refuge dans l'imaginaire. Les digressions qui envahissent le roman brouillent la réalité et ralentissent le déroulement de l'histoire comme si le narrateur cherchait à fuir sa mort inévitable. En outre, si l'imagination permet de transformer le réel, même si c'est de façon momentanée, l'omniscience est aussi le moyen pour les narrateurs de Tarmac et de Gabrielle au bois dormant de justifier une histoire incroyable. Les deux narrateurs cherchent à affirmer que ce qu'ils racontent est vrai, et c'est cette obsession qui sème le doute quant à la vérité de leurs histoires : «On aimait m'entendre dire ce que je pensais, et longtemps j'ai dit la vérité » (Delcourt, p.41), confesse Jacqueline, la seule détentrice de la vérité dans son histoire. "Mes amis regardent autour d'eux, perdus. Que faisait donc Gabrielle la nuit, près de cette vieille maison? Que nous caches-tu, Jacqueline? Que sais-tu?» (Delcourt, p. 164), affirme-t-elle plus 
loin sans qu'on ne sache ce qui est vrai. Le recours à l'omniscience permet donc de soutenir une histoire incroyable.

En conclusion, la subjectivité du point de vue interne et le savoir restreint du narrateur homodiégétique ne permettent pas une ouverture vers un monde plus large que la vie privée alors que, grâce à sa vision et son savoir étendus, le narrateur hétérodiégétique est capable d'aborder plusieurs intrigues et destins simultanément. Pour cette raison, on voit les romanciers étudiés se servir du point de vue omniscient afin d'amoindrir cette différence entre point de vue subjectif du personnage qui vit les événements et point de vue omniscient du narrateur hétérodiégétique. Mais ce choix pour la narration omnisciente ne va pas sans problème. Le narrateur hétérodiégétique et omniscient se caractérise, comme le déduit Genette dans Discours du récit, par sa position qui le tient hors des événements racontés. Voué à l'objectivité, il porte souvent un point de vue distant sur les expériences de son héros. Cette distance entre narration et action a pour effet de dénaturer le caractère réaliste des histoires racontées. Elle rend, en effet, figée la représentation du réel et des faits racontés et altère la signification de l'histoire.

\section{Bibliographie}

BAL, Mieke. (1997), «Narration et focalisation: pour une théorie des instances du récit », Poétique, no 29, p. 107-127. 
DelCourt, Denyse. (2001), Gabrielle au bois dormant, Laval, Éditions Trois, coll. « Topaze».

DiCKNER, Nicolas. (2009), Tarmac, Québec, Alto.

GEnetTE, Gérard. (2007 [1972, dans Figures III, Paris, Seuil, coll. «Poétique »]), Discours du récit, Paris, Seuil, coll. "Points Essais ».

GaILly, Christian. (2004), Dernier amour, Paris, Minuit.

GLOWINSKI, Michal. (1987), "Sur le roman à la première personne », Poétique, no 72, p. 497-507.

Jost, François. (1991), « Pour une narratologie impure », Protée, vol. 19 , no 1 , p. 19-24.

Jouve, Vincent. (2004), «L'autorité textuelle», Enjeux, no 59, p. 143-154.

LinTVELT, Jaap. (1981), Essai de typologie narrative, le "Point de vue » : théorie et analyse, Paris, José Corti.

RABATEL, Alain. (1997), Une histoire du point de vue, Paris / Metz, Klincksieck /Université de Metz.

RIENDEAU, Pascal. (2009), « De la nostalgie d'un monde possible à la possible fin du monde », Voix et Images, vol. 35, noㅜ 1 , p. $120-125$.

Vitoux, Pierre. (1988), «Focalisation, point de vue, perspective », Protée, nำ16, p. 33-38.

KAYSER, Wolfgang. (1977), «Qui raconte le roman?», trad. Anne-Marie Buguet, dans Gérard Genette et Tzvetan Todorov (dir.), Poétique du récit, Paris, Seuil, coll. « Points », p. 59-83. 


\title{
Résumé
}

L'ambiguïté constante qui entoure la notion du point de vue empêche de cerner celle-ci de façon méthodique. Point de vue interne et narrateur homodiégétique vont souvent de pair dans les analyses théoriques. Pourtant, les transgressions de ces constatations théoriques sont nombreuses dans certaines œuvres littéraires, en particulier dans les romans étudiés. Alors que le narrateur homodiégétique, considéré par la majorité des critiques comme une personne, est censé avoir des connaissances subjectives du réel qu'il perçoit, il acquiert, dans ces romans, une vision omnisciente du monde qu'il nous décrit, ce qui rend invraisemblable l'histoire racontée.

\begin{abstract}
First person point of view and homodiegetic narrator are often related in literary theories. But the transgressions of these Narratology concepts are numerous in contemporary literary works, especially the novels studied in this paper. While the homodiegetic narrators, considered by most critics as persons, are supposed to have a limited knowledge of the reality they see, they acquire in the novels an omniscient and unlimited point of view of the world they describe. These transgressions of the first person point of view make the story told hard to believe and require to be analyzed.
\end{abstract}

